\title{
Efecto de los Corticoides Parenterales en la Mortalidad por Covid-19 Severo
}

\section{Effect of Parenteral Corticosteroids in Mortality from Severe Covid-19}

Edinson Dante Meregildo-Rodriguez ${ }^{1,2, a}$, Wilson Marcial Guzmán-Aguilar ${ }^{1,3, b}$, Gustavo Adolfo Vásquez-Tirado ${ }^{1,3, \mathrm{~b}}$, Camilo Peña-Quispe ${ }^{1,3, \mathrm{~b}}$

\begin{abstract}
RESUMEN
Introducción. La infección causada por el SARS-CoV-2 puede producir un cuadro inflamatorio severo, sepsis, falla respiratoria, disfunción orgánica, y muerte. Es plausible fisiopatológicamente, que los corticoides sean una opción terapéutica razonable para Covid-19. Sin embargo, la evidencia del efecto benéfico de los corticoides sobre la mortalidad no es categórica. Objetivo: determinar si existe evidencia que avale el uso de corticoides parenterales en Covid-19 grave y Síndrome de Distrés Respiratorio Agudo (SDRA). Material y método: Este estudio se desarrolló en concordancia con la guía PRIMA. Según la estrategia PICO, nuestra Población fueron los pacientes con Covid-19 grave y/o SRDA; la Intervención, el uso de corticoides parenterales; el Comparador, los pacientes con Covid-19 severo con tratamiento estándar (sin corticoides); y el Outcome, la mortalidad y otros resultados clínicos. La búsqueda se realizó en las bases de datos PubMed, Medline, Cochrane, Embase, y LILACS usando los términos relacionados con nuestra pregunta PICO, entre el de 01 enero al 31 de agosto del 2020. Se logró recopilar un total de 287 artículos, de los que finalmente se seleccionaron 9 que cumplieron con los criterios PICO y de selección. Resultados: Según nuestro estudio, los corticoides parenterales reducen la mortalidad en pacientes con Covid-19 severo y SRDA [OR 0.83 (IC 95\% 0.63-1.08)]. Conclusión: la revisión sistemática muestra que los corticoides parenterales podrían disminuir la mortalidad de pacientes con Covid-19 severo y ARDS.
\end{abstract}

Palabras Clave: Virus del SARS; COVID-19; glucocorticoides; resultado del tratamiento; resultado clínico; mortalidad Fuente: DeCS-BIREME).

\section{ABSTRACT}

Introduction. Infections caused by SARS-CoV-2 may produce a severe inflammatory response, sepsis, respiratory failure, organ dysfunction, and death. From a pathophysiological point of view, it is plausible that glucocorticoids could be a reasonable therapeutic option for Covid-19. However, the evidence supporting the benefit of corticosteroids on mortality and other clinical outcomes is not conclusive. Objective: determinate if there exist evidence supporting corticosteroids therapy in patients with severe Covid19 and Acute Respiratory Distress Syndrome (ARDS). Material and method: This study was performed in concordance with PRISMA guidelines. According to the PICO strategy, Population were patients with severe Covid-19 and/or ARDS; Intervention, parenteral

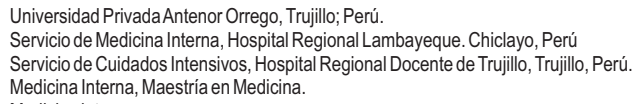

corticosteroid administration; Comparator, patients with severe Covid-19 and/or ARDS with standard treatment (without corticosteroids); and Outcome, mortality and other clinical outcomes. Search strategy was performed in the data bases PubMed, Medline, Cochrane, Embase, and LILACS, using terms related with the PICO question; from January 1 to august 31 (2020). A total of 287 articles were initially retrieved. Of these, we finally selected only 9 article which fulfilled with PICO and selection criteria. Results: According to our study, parenteral corticosteroids reduce mortality in patients with Severe Covid-19 and/or ARDS [OR 0.83 (IC 95\% 0.63-1.08)]. Conclusion: the systematic review shows that parenteral corticosteroids could reduce the mortality of patients with severe Covid-19 and ARDS.

Keywords: SARS Virus; COVID-19; glucocorticoids; treatment outcome; clinical outcome; mortality (Source: DeCS-BIREME). 
Los coronavirus son un grupo de patógenos que afecta a animales y humanos. Al final del 2019, en Wuhan, China, se identificó un virus que producía una neumonía severa. Esta enfermedad se propagó por toda Asia, afectando posteriormente a otros continentes. En febrero del 2020, la Organización Mundial de la Salud designó a esta enfermedad como Covid-19, y al virus causante de esta enfermedad como coronavirus 2 del síndrome respiratorio agudo severo (SARS-CoV-2) $)^{(1,2)}$. La transmisión a través de aerosoles, es un mecanismo importante de diseminación de la enfermedad ${ }^{(3)}$. El SARS-CoV-2 se ha detectado en muestras no respiratorias como heces, sangre, secreciones oculares y semen; sin embargo, su rol en la transmisión es incierto ${ }^{(4,5)}$.

EI SARS COV-2 puede producir una enfermedad grave en un $5 \%$ de infectados. Estos pacientes requieren ingreso en la unidad de cuidados intensivos (UCI). Los principales predictores para requerir ingreso a $\mathrm{UCl}$ son: saturación de oxígeno $<92 \%$ con oxígeno ambiental, linfopenia, edad $\geq 60$ años y obesidad. Otros factores de riesgo son: hipertensión arterial, enfermedad renal crónica, y diabetes mellitus. Se consideran como criterios de gravedad los niveles de dímero $D$ superiores a $1000 \mathrm{ng} / \mathrm{ml}$ y una puntuación de SOFA elevada al ingreso $0^{(6,7)}$.

La enfermedad producida por el virus SARS CoV-2, puede producir un cuadro inflamatorio severo, que puede llevar a falla respiratoria, sepsis, disfunción de órganos y muerte ${ }^{(8)}$. Por lo que se espera que los medicamentos antiinflamatorios, como los corticoides, sean una alternativa terapéutica. Sin embargo, la evidencia aún no es categórica ${ }^{(9-23)}$. En diversos estudios recientes, incluso meta análisis, se han extrapolado resultados de otras enfermedades virales, e incluso bacterianas, a la Covid-19 $9^{(25-28)}$. El presente estudio buscó determinar si existe evidencia que avale el uso de corticoides parenterales en pacientes con Covid-19 grave. Según la estrategia PICO, nuestra Población de estudio fueron los pacientes con Covid-19 grave y/o SRDA; la Intervención a evaluar es el uso de corticoides parenterales; el Comparador, los pacientes con infección Covid-19 que recibieron tratamiento estándar; y el Outcome, la mortalidad en estos pacientes.

\section{MATERIAL Y MÉTODOS}

Estrategia de búsqueda: nuestro estudio se llevó en concordancia con la guía PRISMA. La búsqueda se realizó en las bases de datos PubMed, Medline, Cochrane, Embase, y LILACS usando los términos relacionados con nuestra pregunta PICO; entre el de 01 enero al 31 de agosto del 2020. Complementariamente, se realizó una búsqueda secundaria de artículos no incluidos en dichas bases. La expression de búsqueda fue: ("coronavirus" OR "COVID-19", OR "2019-nCoV", OR "SARS-COV-2" OR "Acute Respiratory Distress
Syndrome"), AND ("corticosteroids" OR "glucocorticoids", "hydrocortisone" OR "methylprednisolone" OR "dexamethasone" OR "steroid"), AND ("mortality" OR "mechanical ventilation" OR "ICU admission" OR "ICU stay" OR "hospital stay").

Criterios de elegibilidad. Incluimos sólo estudios primarios [ensayos clínicos (ECs), estudios de cohorte y estudios transversales] que evaluaron corticoides parenterales (dexametasona, metilprednisolona, hidrocortisona, prednisolona) en pacientes adultos con Covid-19 grave y/o SDRA y su efecto en la mortalidad.

Selección de los estudios. Se recopiló artículos primarios y secundarios los cuales cumplieron con los criterios PICO y de selección para el análisis cuantitativo.

Valoración de sesgo. Para los estudios de cohorte y transversales se usó la herramienta Newcastle-Ottawa Scale (NOS); y para los ensayos clínicos aleatorizados, el RoB2 (herramienta de Cochrane).

Extracción de datos y análisis. Para cada publicación seleccionada se extrajo: primer autor; año y mes de publicación; país; tamaño de muestra; covariables relacionadas; tipo, dosis y duración del tratamiento con corticosteroides. La medida de resultado fue el efecto en la mortalidad [RR, OR, HR, e intervalo de confianza (IC) al 95\%].

Heterogeneidad. Evaluamos heterogeneidad clínica, metodológica y estadística. La heterogeneidad clínica se evaluó a través de diferencias entre pacientes, tratamientos o desenlaces. La heterogeneidad metodológica, según variabilidad en los diseños, medidas del efecto y control de sesgos. La heterogeneidad estadística se estimó con la determinación de un gráfico forest plot, donde se valoró el efecto de los corticoides a favor, o en contra, del resultado. En el gráfico forest plot, visualmente consideramos que existe poca heterogeneidad cuando los estimados de los estudios se ubican cercanamente entre sí y superponen sus ICs. Consideramos como heterogeneidad significativa un $12 \geq 60 \%$, y según el estadístico Chi-cuadrado un $\mathrm{p}$ valor $\leq 0,05$. Dada, la significativa heterogeneidad en el forest plot global (considerando todos los estudios) (I2 84\% y $p<0,00001$ ), estimamos modelos de efectos aleatorios y la prueba Tau2. Sin embargo, cuando analizamos solos los ECAs (excluyendo estudios de cohortes y transversales), obtuvimos que una heterogeneidad estadística no significativa (I2 $44 \%$ y $\mathrm{p}=0,17)$ por lo que usamos el modelo de efectos fijos.

\section{RESULTADOS}

Tras la búsqueda realizada, en las bases de datos mencionadas, durante el 01 de enero al 31 de agosto del 


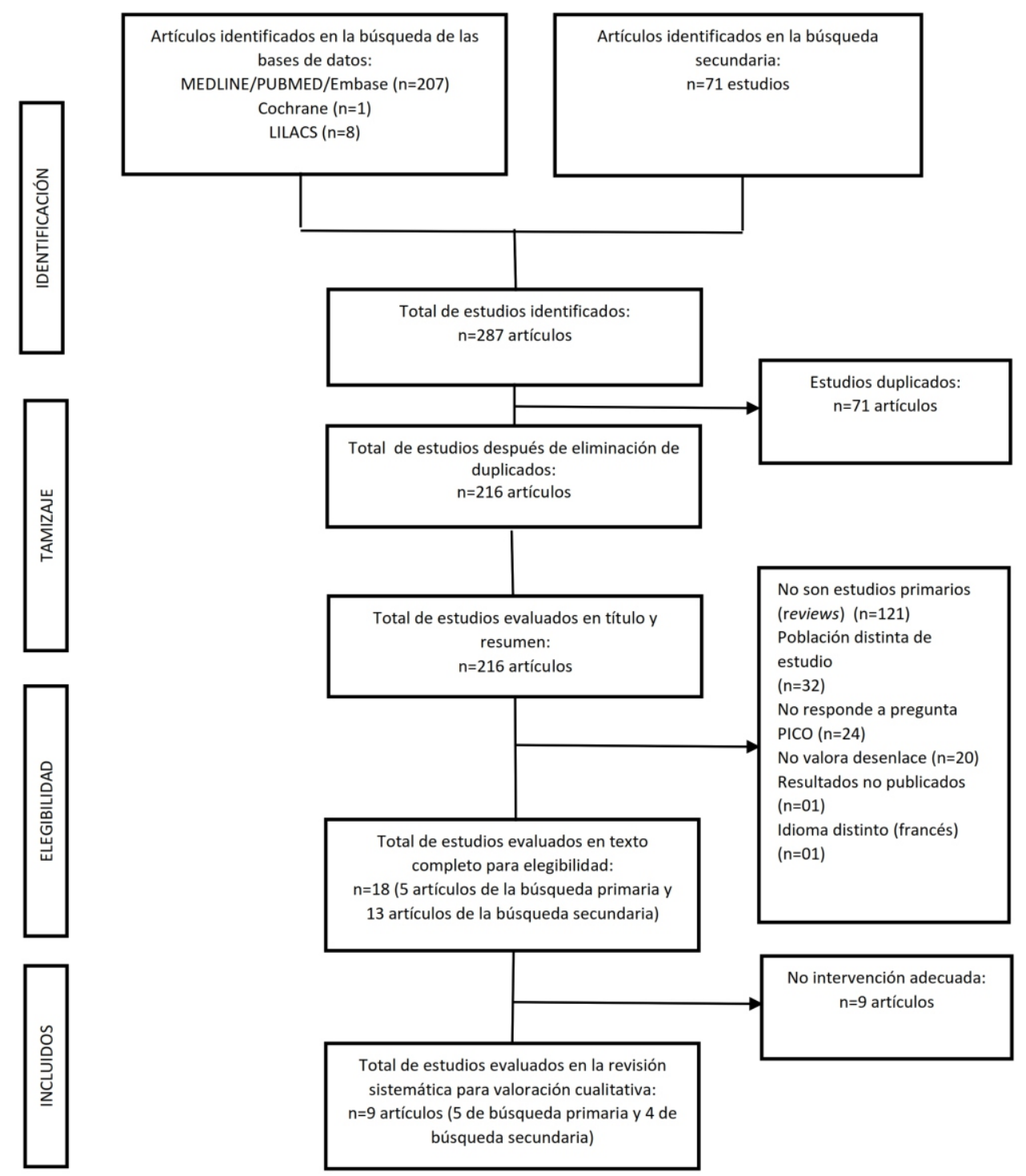

Figura 1

Diagrama de flujo PRISMA de la selección de los estudios primarios sobre el uso de corticoide en pacientes con infección por SARS- CoV-2.

2020. Se logró recopilar un total de 287 artículos relacionados con la pregunta PICO (Figura 1). De éstos, 216 artículos se obtuvieron de la búsqueda primaria y 71 de la búsqueda secundaria. Se realizó una revisión por pares, quedando 18 artículos. De estos artículos, finalmente se seleccionaron 9 , los cuales cumplieron con los criterios PICO y de selección. Se excluyeron: cartas al editor, serie de casos, correspondencias y revisiones narrativas. Del total de 9 artículos, dos fueron estudios transversales ${ }^{(14,19)}$, cuatro cohortes retrospectivas ${ }^{(11,13,16,20)}$, y tres fueron ensayos clínicos aleatorizados $^{(21-23)}$. Los 2 estudios transversales se realizaron en China; y de los estudios de cohorte, 3 se realizaron en China ${ }^{(13,16,21)}$ y uno en Estados Unidos de América ${ }^{(11)}$. Los estudios observacionales (transversales y cohortes), se caracterizaron por amplias diferencias en el número de participantes (entre 31 a 416), severidad de la enfermedad; tipo, dosis y tiempo del corticoide administrado). En todos ellos se usó equivalente metilprednisolona en un rango de dosis de 0,5 a $3 \mathrm{mg} / \mathrm{kg} / \mathrm{d}^{(11,13,16,21)}$ (Tabla 1$)$.

Los ensayos clínicos (EC) se realizaron en 3 países: Reino Unido ${ }^{(21)}$, España ${ }^{(22)}$ y Brasil $^{(23)}$. El primero fue un EC aleatorizado, controlado, de etiqueta abierta y adaptativo en 6425 pacientes en total diagnosticados al 
Tabla 1. Características generales de los estudios incluidos sobre el uso de corticoide en pacientes con infección severa por SARS-CoV-2. con infección por SARS- CoV-2.

\begin{tabular}{|c|c|c|c|c|c|c|c|c|}
\hline Autor & Lugar & $\begin{array}{l}\text { Seguimiento } \\
\text { (dias) }\end{array}$ & Tipo de estudio & $\begin{array}{l}\text { Diagnóstico de } \\
\text { Covid-19 }\end{array}$ & $n$ & $\begin{array}{l}\text { Estadio } \\
\text { Severidad }\end{array}$ & Corticoide & Dosis y duración \\
\hline Raef $F(11)$ & EUA & 15 & Cohorte prospectiva & RT-PCR & 213 & $\begin{array}{c}\text { Moderado / } \\
\text { Severo }\end{array}$ & MTP & $0.5-1 \mathrm{mg} / \mathrm{k}, 3$ días \\
\hline Wang D (13) & China & 40 & Cohorte retrospectiva & RT-PCR & 115 & $\begin{array}{l}\text { Critico / } \\
\text { No Critico }\end{array}$ & MTP & $\begin{array}{l}\text { Grupo A: } 0.5-1 \mathrm{mg} / \mathrm{kg} ; 3 \text { días } \\
\text { Grupo B: } 1-3 \mathrm{mg} / \mathrm{kg} ; 3 \text { a } 10 \text { días }\end{array}$ \\
\hline Shang J (14) & China & 60 & $\begin{array}{c}\text { Transversal retrospectivo } \\
\text { multicéntrico }\end{array}$ & RT-PCR & 416 & $\begin{array}{l}\text { No Severo / } \\
\text { Critico }\end{array}$ & MTP & $\begin{array}{l}\text { Sobreviviente Severo: } 38.7 \mathrm{mg} \text {; } 2 \text { días. } \\
\text { Sobreviviente no severo: } 40 \mathrm{mg} ; 2 \text { días. } \\
\text { Muerto: } 65 \mathrm{mg} ; 6 \text { días }\end{array}$ \\
\hline Wang $Y(16)$ & China & 35 & Cohorte retrospectiva & RT-PCR & 46 & Severo & MTP & $1-2 \mathrm{mg} / \mathrm{k} / \mathrm{d} ; 5$ días \\
\hline Zha L (19) & China & 30 & Transversal & RT-PCR & 31 & Severo & MTP & $40-80 \mathrm{mg} / \mathrm{d} ; 5$ días \\
\hline Horby P (20) & $\begin{array}{l}\text { Reino } \\
\text { Unido }\end{array}$ & 28 & ECA abierto & RT-PCR & 6425 & $\begin{array}{c}\text { Con 02; Sin 02; Con } \\
\text { VMI }\end{array}$ & DXM & $6 \mathrm{mg} / \mathrm{d} ; 10$ días \\
\hline Xu J (21) & China & 60 & $\begin{array}{c}\text { Cohorte retrospectiva } \\
\text { multicéntrico }\end{array}$ & RT-PCR & 239 & Severo / SDRA & MTP & $60.9 \pm 21.7 \mathrm{mg}$ de 3 a 10 días \\
\hline $\begin{array}{l}\text { Jeronimo } \\
\text { CMP (22) }\end{array}$ & Brazil & 28 & $\begin{array}{l}\text { EC randomizado, doble ciego, } \\
\text { controlado con placebo fase lib }\end{array}$ & RT-PCR & 393 & Severo / Moderado & MTP & $\begin{array}{l}0.5 \mathrm{mg} / \mathrm{Kg} / 12 \mathrm{~h} \text { por } 5 \text { dias. Además, } \\
\text { hidrocortisona para pacientes con shock }\end{array}$ \\
\hline $\begin{array}{l}\text { Corral- } \\
\text { Gudino (23) }\end{array}$ & España & No precisa & $\begin{array}{c}\text { EC parcialmente randomizado, } \\
\text { etiqueta abierta }\end{array}$ & $\begin{array}{l}\text { No precisa método } \\
\text { diagnóstico }\end{array}$ & 85 & $\begin{array}{c}\text { Covid-19 con riesgo } \\
\text { de SDRA }\end{array}$ & MTP & $\begin{array}{c}40 \mathrm{mg} / 12 \mathrm{~h}, 3 \text { días; luego } 20 \mathrm{mg} / 12 \mathrm{~h}, 3 \\
\text { días. }\end{array}$ \\
\hline
\end{tabular}

VMI: Ventilación mecánica invasiva. SDRA: Síndrome de Dificultad Respiratoria Agudo. MTP: metilprednisolona. DXM: dexametasona. EC: ensayo clínico.

Tabla 2. Características de los estudios incluidos sobre el uso de corticoide en pacientes con infección por SARS- CoV-2 y el efecto en la mortalidad o supervivencia.

\begin{tabular}{|c|c|c|c|c|c|c|c|c|c|}
\hline Autor & $\begin{array}{l}\text { Total con } \\
\text { corticoide }\end{array}$ & $\begin{array}{l}\text { Total sin } \\
\text { corticoide }\end{array}$ & $\begin{array}{l}\text { Muertos con } \\
\text { corticoide }\end{array}$ & $\begin{array}{l}\text { Muertos sin } \\
\text { corticoide }\end{array}$ & $\begin{array}{l}\text { Vivos con } \\
\text { corticoide }\end{array}$ & $\begin{array}{l}\text { Vivos sin } \\
\text { corticoides }\end{array}$ & Medida de efecto & IC y significancia & $\mathrm{p}$ \\
\hline Raef F (11) & 132 & 81 & 18 & 21 & 114 & 60 & OR: 0.45 & IC [0.22-0.91] & $\mathrm{p}=0.024$ \\
\hline Wang $D$ (13) & 73 & 42 & $\begin{array}{r}24 \text { (muerte o } \\
\text { ingreso a UCI) }\end{array}$ & $\begin{array}{l}5 \text { (muerte o } \\
\text { ingreso a } \\
\text { UCI) }\end{array}$ & $\begin{array}{l}49 \text { (no } \\
\text { muerte o } \\
\text { ingreso a } \\
\text { UCI) }\end{array}$ & $\begin{array}{l}37 \text { (no } \\
\text { muerte o } \\
\text { ingreso a } \\
\text { UCl) }\end{array}$ & OR: 2.155 & 0.493-9.427 (95\% Cl) & $p=0.308$ \\
\hline Shang J (14) & 196 & 220 & 43 & 8 & 153 & 67 & $\begin{array}{l}\text { OR: } 2.3538 \text { [Calculado a } \\
\text { partir de datos del estudio] }\end{array}$ & $\begin{array}{c}1.0498-5.2776(95 \% \mathrm{Cl}) \\
\text { [Calculado a partir de datos del } \\
\text { estudio] }\end{array}$ & $\begin{array}{l}\text { Sobreviviente Severo/Crítico } \\
\text { vs. Muerto } p<.05 ; \\
\text { Sobreviviente Común (No } \\
\text { Severo/No crítico) vs. } \\
\text { Sobreviviente Severo/Crítico } \\
\quad p<.05\end{array}$ \\
\hline Wang $Y(16)$ & 26 & 20 & 2 & 1 & 24 & 19 & $\begin{array}{l}\text { OR: 1.5833. [Calculado a } \\
\text { partir de datos del estudio] }\end{array}$ & $\begin{array}{c}0.1333-18.8090(95 \% \mathrm{Cl}) \\
\text { [Calculado a partir de datos del } \\
\text { estudio] }\end{array}$ & $\begin{aligned} \mathrm{p}= & 0.7159 \text { [Calculado a partir } \\
& \text { de datos del estudio] }\end{aligned}$ \\
\hline Zha L (19) & 11 & 20 & 0 & 0 & 11 & 20 & $\begin{array}{l}\text { OR (1.7826) [Calculo con los } \\
\text { datos del estudio]. }\end{array}$ & $95 \%$ Cl: 0.0331 - 95.9699 & $p=0.7762$ \\
\hline Horby P (20) & 2558 & 5386 & 454 & 1065 & 2104 & 4321 & \multicolumn{3}{|c|}{$\begin{array}{l}\text { La dexametasona redujo la mortalidad en } 1 / 3 \text { en los pacientes con VMI }(29.0 \% \text { vs. } 40.7 \% \text {, RR } \\
0.65[95 \% \mathrm{Cl} 0.51-0.82] ; p<0.001) \text {, y en } 1 / 5 \text { en los pacientes con } 02 \sin \text { VMI }(21.5 \% \text { vs. } 25.0 \% \text {, } \\
\text { RR } 0.80 \text { [95\% } \mathrm{Cl} 0.70-0.92] ; \mathrm{p}=0.002) \text {. No redujo mortalidad en pacientes que no requirieron } \\
\mathrm{O} 2(17.0 \% \text { vs. } 13.2 \%, \mathrm{RR} 1.22[95 \% \mathrm{Cl} 0.93-1.61] ; \mathrm{p}=0.14) \text {. }\end{array}$} \\
\hline Xu J (21) & 189 & 50 & 118 & 29 & 71 & 21 & No definida & No definida & $\mathrm{p}=0.339$ \\
\hline $\begin{array}{l}\text { Jeronimo } \\
\text { CMP (22) }\end{array}$ & 194 & 199 & 72 & 76 & 122 & 123 & \multicolumn{3}{|c|}{$\begin{array}{l}\text { No hubo diferencias en la mortalidad a los } 28 \text { días entre los grupos de comparación: } \\
\text { Metilprednisolona a razón de } 0.5 \mathrm{mg} / \mathrm{kg} \text { dos veces al día versus solución salina por } 5 \text { días. HR } \\
(95 \% \mathrm{Cl}) \text { / Diferencia absoluta }(95 \% \mathrm{Cl}): 0.924(0.669-1.275) \text { con un p de } 0.629 \text {. } \\
\text { En el análisis de subgrupo hubo diferencias en la mortalidad a favor de la metilprednisolona en } \\
\text { los mayores de } 60 \text { años. HR:0.634 }(0.411-0.978) \text {. } \mathrm{p}=0.039\end{array}$} \\
\hline $\begin{array}{l}\text { Corral- } \\
\text { Gudino (23) }\end{array}$ & 56 & 29 & 19 & 14 & 37 & 15 & \multicolumn{3}{|c|}{$\begin{array}{l}\text { Análisis por intención a tratar para Outcome Primario Compuesto (admisión a UCI, ventilación } \\
\text { no invasiva, o muerte) RR } 0.55(0.33-0.91), p=0.025 \\
\text { Análisis por protocolo para Outcome Primario Compuesto (admisión a UCI, ventilación no } \\
\text { invasiva, o muerte) RR } 0.37 \text { ( } 0.19-0.74), p=0.0037\end{array}$} \\
\hline
\end{tabular}

UCI: Unidad de Cuidados Intensivos. OR: Odds Ratio. RR: Risk Ratio. HR: Hazzard Ratio. 
Tabla 3. Evaluación de sesgo de los estudios incluidos sobre el uso de corticoide en pacientes con infección por SARS- CoV-2.

\begin{tabular}{|c|c|c|c|c|c|c|c|c|}
\hline Articulo evaluado & Autor & Diseño & Herramienta & Selección (4) & $\begin{array}{c}\text { Comparabili } \\
\text { dad (2) }\end{array}$ & $\begin{array}{l}\text { Desenlace } \\
\text { (3) }\end{array}$ & Total & Conclusión \\
\hline $\begin{array}{l}\text { Early Short Course Corticosteroids in Hospitalized Patients } \\
\text { with COVID-19. }\end{array}$ & Raef F (11) & Cohorte prospectiva & NOS & $* \star \star$ & $* \star$ & * & 6 & Alto Riesgo \\
\hline $\begin{array}{l}\text { No Clear Benefit to the Use of Corticosteroid as Treatment } \\
\text { in Adult Patients with Coronavirus Disease 2019: A } \\
\text { Retrospective Cohort Study }\end{array}$ & $\begin{array}{l}\text { Wang D } \\
(13) \text {. }\end{array}$ & Cohorte retrospectiva & NOS & ** & ** & $* * \star$ & 7 & Bajo Riesgo \\
\hline $\begin{array}{l}\text { The Treatment and Outcomes of Patients with COVID-19 in } \\
\text { Hubei, China: A Multi-Centered, Retrospective, } \\
\text { Observational Study }\end{array}$ & $\begin{array}{l}\text { Shang J } \\
(14) \text {. }\end{array}$ & $\begin{array}{l}\text { Transversal } \\
\text { multicéntrico- } \\
\text { retrospectivo }\end{array}$ & NOS & $* *$ & $* *$ & * & 5 & Alto Riesgo \\
\hline $\begin{array}{l}\text { Early, low-dose and short-term application of corticosteroid treatment in } \\
\text { patients with severe COVID-19 pneumonia: single-center experience from } \\
\text { Wuhan, China }\end{array}$ & $\begin{array}{l}\text { Wang } Y \\
(16) \text {. }\end{array}$ & Cohorte retrospectiva & NOS & $* \star \star$ & $* *$ & * & 6 & Alto Riesgo \\
\hline $\begin{array}{l}\text { Corticosteroid treatment of patients with coronavirus } \\
\text { disease } 2019 \text { (COVID-19) }\end{array}$ & Zha L (19). & Transversal & NOS & * & $* *$ & * & 5 & Alto Riesgo \\
\hline $\begin{array}{l}\text { Clinical course and predictors of } 60 \text {-day mortality in } 239 \\
\text { critically ill patients with COVID-19: a multicenter } \\
\text { retrospective study from Wuhan, China. }\end{array}$ & Xu J (21). & Cohorte retrospectiva & NOS & $* * * *$ & * & * & 6 & Alto Riesgo \\
\hline $\begin{array}{l}\text { Effect of Dexamethasone in Hospitalized Patients with } \\
\text { COVID-19 - Preliminary Report. }\end{array}$ & $\begin{array}{l}\text { Horby Pl } \\
\text { (20). }\end{array}$ & EC adaptativo & RoB2 & \multicolumn{5}{|c|}{$\begin{array}{c}\text { Bajo Riesgo Global, pero Con Algunas Preocupaciones en el Primer } \\
\text { Dominio }\end{array}$} \\
\hline $\begin{array}{l}\text { Methylprednisolone as Adjunctive Therapy for Patients } \\
\text { Hospitalized With COVID-19 (Metcovid): A Randomised, } \\
\text { Double-Blind, Phase IIb, Placebo-Controlled Trial }\end{array}$ & $\begin{array}{l}\text { Jeronimo } \\
\text { CMP (22) }\end{array}$ & $\begin{array}{l}\text { EC randomizado, doble } \\
\text { ciego, controlado con } \\
\text { placebo fase lib }\end{array}$ & RoB2 & \multicolumn{5}{|c|}{$\begin{array}{c}\text { Bajo Riesgo Global, pero Con Algunas Preocupaciones en el Primer } \\
\text { Dominio }\end{array}$} \\
\hline $\begin{array}{l}\text { GLUCOCOVID: A controlled trial of methylprednisolone in } \\
\text { adults hospitalized with COVID-19 pneumonia }\end{array}$ & $\begin{array}{l}\text { Corral- } \\
\text { Gudino (23) }\end{array}$ & $\begin{array}{l}\text { EC parcialmente } \\
\text { randomizado, etiqueta } \\
\text { abierta }\end{array}$ & RoB2 & \multicolumn{5}{|c|}{$\begin{array}{c}\text { Bajo Riesgo Global, pero Con Algunas Preocupaciones en el Primer } \\
\text { Dominio }\end{array}$} \\
\hline
\end{tabular}

NOS: Score Newcastle-Ottawa para evaluación de estudios observacionales. RoB2: Herramienta de Cochrane para evaluación de riesgo de sesgo de ensayos clínicos. EC: Ensayos Clínicos.

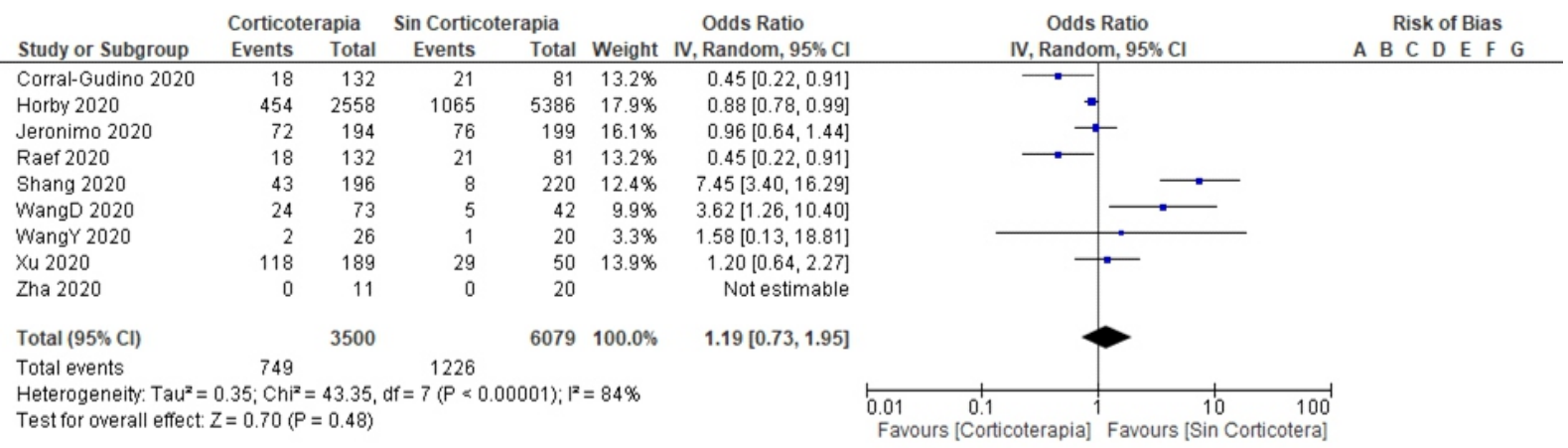

*En el análisis se eliminó el estudio de Zha et al (19) ya que al haber eventos no aporta estadísticamente, y considerando efectos aleatorios puesto que 12 es $\geq 60 \%$.

\section{Figura 2}

Forest plot global (incluyendo estudios transversales, cohortes y ensayos clínicos) sobre efecto de los corticoides sistémicos en la mortalidad de pacientes Covid-19 severo*

$100 \%$ con PCR-TR; en una proporción aproximada de 1:2 entre el grupo intervención y control; período de seguimiento de 28 días para medir el outcome combinado de mortalidad y necesidad de ventilación mecánica invasiva (VMI) con el uso de dexametasona
$6 \mathrm{mg} / \mathrm{d}$ por 10 días, la cual resulto a favor de disminución del efecto combinado de manera significativa ${ }^{(21)}$. GLUCOCOVID fue un EC, parcialmente aleatorizado, etiqueta abierta, adaptativo, contempló análisis por intención a tratar (ITT) y por protocolo (PP) 


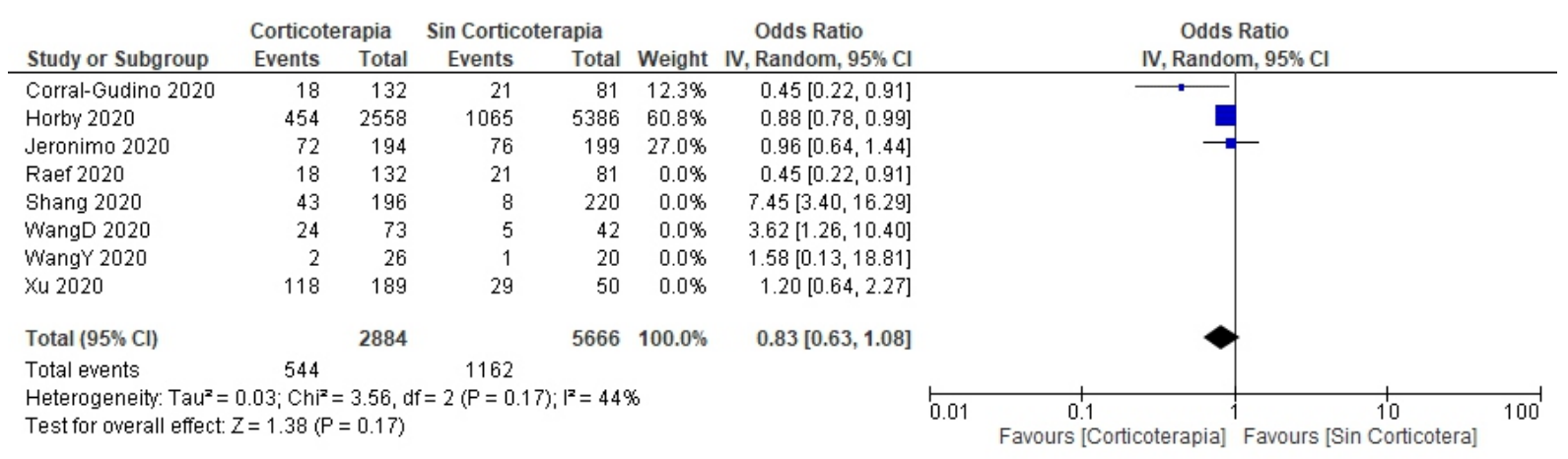

**El análisis incluyó solo 3 estudios (21-23) considerando efectos fijos, puesto que 12 es $<60 \%$.

Figura 3

Forest plot que incluye solo ensayos clínicos (excluyendo estudios transversales y cohortes) sobre efecto de los corticoides sistémicos en la mortalidad de pacientes Covid-19 severo**

en 85 pacientes en total, diagnosticados al $100 \%$ con PCR-TR en una en un proporción aproximada de 2:1 entre el grupo intervención y control; para medir el outcome combinado de mortalidad por todas las causas, necesidad de UCI o VMI con el uso de metilprednisolona $40 \mathrm{mg} / \mathrm{d}$ por 3 días seguido de 20 $\mathrm{mg} / \mathrm{d}$ por días, la cual no resultó en efecto benéfico en los outcomes combinados ${ }^{(2)}$. El METACOVID fue un EC aleatorizado (ECA), doble ciego, controlado con placebo fase IIb por ITT en 393 pacientes en total diagnosticados al $81,3 \%$ con PCR-TR en una proporción de 1:1; con 28 días de seguimiento y determinación de mortalidad a los 7, 14 y 28 días; lo cual no resultó en efecto en el outcome primario de mortalidad con metilprednisolona $0.5 \mathrm{mg} / \mathrm{kg} / \mathrm{d}$ por 5 día comparado con solución salina ${ }^{(23)}$ (Tabla 1). La proporción de pacientes expuestos y no expuestos entre los estudios no fue uniforme. La medida del efecto en la mortalidad usado fue el OR e IC 95\% para los estudios de cohorte, y RR en los 2 primeros EC y HR en el tercero (Tabla 2).

En la evaluación de sesgos se usó la herramienta NOS para cohortes; y para ECAs, se usó el ROB2. Tres de cuatro estudios de cohortes, y los dos estudios trasversales tuvieron riesgo alto de sesgo. Todos los ECAs tuvieron bajo riesgo de sesgo. Al determinar la heterogeneidad clínica y metodológica, en los estudios observacionales y de cohortes resultó alta; y baja en ECAs (Tabla 3). Al determinar el forest plot global (estudios de cohortes, transversales, y ECAs) con efectos aleatorios, la heterogeneidad estadística resultó alta sin efecto en la mortalidad de los corticoides sistémicos. Sin embargo, al analizarlos por separados, sólo los ECAs, con estimación de efectos fijos la heterogeneidad estadística resultó $<50 \%$ y mostró que los corticoides tienen efecto en la disminución de la mortalidad de manera significativa. Los estudios observacionales y de cohortes tuvieron heterogeneidad clínica, metodológica y estadística alta, sin efecto en la mortalidad (Figura 2 y 3 ).

\section{DISCUSIÓN}

De acuerdo a nuestros resultados, los corticoides parenterales probablemente tienen un efecto benéfico sobre la mortalidad en pacientes con Covid-19 severo y SRDA [OR 0,83 (IC 95\% 0,63-1,08)].

Aunque algunos estudios primarios analizados en nuestra revisión incluyeron outcomes secundarios independientes o combinados (admisión a $\mathrm{UCI}$, ventilación no invasiva, con mortalidad), nosotros no realizamos un metaanálisis de outcomes secundarios, dada la heterogeneidad de estas variables en los estudios incluidos.

Nuestros resultados concuerdan con las conclusiones de otras revisiones sistemáticas y metaanálisis (RSyMETA) previamente publicadas ${ }^{(24,25)}$, pero se contraponen con otros estudios $^{(26-28)}$. Sin embargo, algunos de los estudios primarios incluidos en estos estudios discordantes con el nuestro se caracterizaron por su tener alto riesgo de sesgo y una gran heterogeneidad. Siemieniuk et al., incluyeron solo pacientes con SARS-CoV-2, y analizaron dos de los estudios ${ }^{(21,22)}$ que nosotros también incluimos en nuestro análisis, y concluyeron que los corticoides probablemente reducen la mortalidad y la necesidad de ventilación mecánica en pacientes con Covid-19 ${ }^{(24)}$. Ye et al., incluyeron varios estudios con etiologías diversas (Covid-19, otros coronavirus, e influenza); solo dos de estos estudios incluyeron pacientes con Covid-19, en uno de los cuales no se precisa dosis, duración, ni tipo de corticoide. Los autores concluyeron que, a pesar que la calidad de la evidencia es muy baja, los corticoides podrían reducir la mortalidad en pacientes con Covid19 y SDRA ${ }^{(25)}$. Lee y col., revisaron estudios con pacientes infectados con SARS, MERS y Covid-19; en los dos únicos estudios que incluyeron pacientes con Covid19 , no se precisa dosis ni duración del corticoide administrado. Sin embargo, a pesar que este 
metaanálisis no mostró diferencias en términos de mortalidad, los autores concluyeron que se debería considerar el uso de corticoides en las infecciones por coronavirus, incluyendo Covid-19 ${ }^{(27)}$. Por el contrario, Yang $Z$ et al., incluyendo estudios con etiologías diversas (SARS-CoV-2, SARS-CoV, MERS-CoV); aunque en ninguno de los dos estudios que se examinó pacientes con Covid-19 se especifica dosis ni duración del corticoide, se concluyó que los corticosteroides se asocian con incremento de la mortalidad en pacientes con neumonía por coronavirus ${ }^{(26)}$. Similar resultado mostró el estudio de $\mathrm{Li}$ et al., donde incluyeron pacientes con SARS-CoV-2, SARS-CoV, y MERS-CoV; sin embargo, en ninguno de los cuatro estudios primarios que incluyó pacientes con Covid-19 se especifica dosis ni duración del corticoide empleado. Los autores concluyeron que los corticoides en pacientes con infección por SARS-CoV-2, SARS-CoV, y MERS-CoV no mejoran supervivencia, no reduce la estancia hospitalaria, la necesidad de ingreso a UCl o de $\mathrm{VMI}^{(28)}$.

Principales fortalezas. Encontramos cinco RSyMETs publicadas hasta la actualidad, que incluyeron al menos un estudio primario sobre corticoides y Covid-19 ${ }^{(24-28)}$. Casi todas estas publicaciones incluyeron también otros coronavirus, e incluso influenza ${ }^{(25-27)}$. Así mismo, en varios de los estudios primarios incluidos, no se precisa el tipo de corticoide empleado, la dosis, ni duración de la administración ${ }^{(25-27)}$. Si consideramos, sólo estudio primarios sobre SARS-CoV-2 y corticoides, cuatro de estas RSyMETs ${ }^{(25-27)}$ incluyeron sólo dos estudios primarios cada una, y una de ellas incluyó cuatro estudios primarios ${ }^{(24)}$ sobre SARS-CoV-2 y corticoides.

Nuestro estudio incorpora estudios más recientes, más pacientes, y sólo incluye estudios primarios que únicamente incluyeron pacientes con SARS-CoV-2 y que especificaron el tipo, dosis, y duración de administración de corticoides. Todos esto, proporciona evidencia más robusta respecto al efecto de los corticoides sistémicos sobre la mortalidad en pacientes con Covid-19 severo y SDRA. Así mismo, nosotros empleamos una estrategia de búsqueda muy amplia y sensible, pero sólo incluimos estudios revisados por pares, y excluimos aquellos publicados como preprints. Otra Fortaleza de nuestro estudio es que investigadores independientes (tutores) supervisaron la extracción artículos, el análisis crítico de los mismo, y el análisis de sesgos.

Principales limitaciones. Diseño de estudio y análisis. Esta revisión tiene importantes limitaciones, principalmente respecto a la calidad variable de la evidencia disponible y la heterogeneidad. La mayoría de estudios incluidos son retrospectivos; lo cual limita la capacidad para evaluar variables confusoras y resultados clínicos. Por ejemplo, en la mayoría de estudios retrospectivos no realizaron el ajuste de variables confusoras importantes como la gravedad y grado de comorbilidad del paciente, uso de múltiples medicamentos -además de corticoides-, algunos de los cuales actualmente han sido suspendidos en el tratamiento del paciente con Covid-19 por futulidad terapéutica o por aparente incremento de ventos adversos. Así mismo, se ha reportado en varios estudios, que los pacientes de mayor gravedad, suelen recibir con más frecuencia corticoterapia. Es muy probable que todos estos factores hayan influido sobre el outcome reportado, y el efecto de los corticoides sobre la mortalidad haya sido infra o supra estimado.

Heterogeneidad de resultados. En el análisis acumulado se tomó en cuenta el modelo de efectos aleatorios para explicar la heterogeneidad. La elevada heterogeneidad entre los estudios es explicada en parte por la falta de control confusores y diferencias en tamaño de muestras entre los estudios. Así mismo, al análisis por funnel plot sugería un sesgo de publicación, aunque, debido a que finalmente solo incluimos nueve estudios este tipo de este método no es el más adecuado para evaluar este tipo de sesgo.

Importancia para la salud pública y necesidad de mayor investigación. Nuestros hallazgos podrían tener implicancias en términos de salud pública e influir en las decisiones individuales del clínico tratante. Esto resulta particularmente relevante si consideramos que la pandemia del de Covid-19 continúa en expansión. De acuerdo a la gran heterogeneidad de la evidencia actualmente disponible, y los resultados dispares en relación al beneficio de los corticoides sistémicos sobre mortalidad por Covid-19 severo, resulta evidente que se requieren más estudios con mejor diseño y que tomen en cuenta las limitaciones detectadas en esta y revisiones previas. A diferencia de otras revisiones sistemáticas y meta análisis previos, nuestro estudio incorpora un mayor número de estudios más recientes, solamente incluimos estudios primarios que exclusivamente consideraron pacientes con SARS-CoV2 , y que precisaron el tipo, dosis, y duración de la administración del corticoide.

La revisión sistemática muestra que los corticoides parenterales podrían disminuir la mortalidad de pacientes con Covid-19 severo y ARDS.

Conflictos de interés: Los autores niegan conflictos de interés.

Financiamiento: Autofinanciado.

Agradecimientos: A nuestros maestros y tutores, los Doctores: Cesar Augusto Ugarte Gil y Lely Del Rosario Solari Zerpa.

\section{REFERENCIAS BIBLIOGRÁFICAS}

1. Gorbalenya AE, Baker SC, Baric RS, et al. Severe 
acute respiratory syndrome-related coronavirus: The species and its viruses - a statement of the Coronavirus Study Group. bioRxiv 2020. https://www. biorxiv.org/content/10.1101/2020. 02.07.937862v1 (Accessed on June 12, 2020).

2. Tang $X$, Wu C, Li X, Song Y, Yao X, Wu X, Duan Y, Zhang H, Wang Y, Qian Z, Cui J, Lu J. On the origin and continuing evolution of SARS-CoV-2. Natl Sci Rev. 2020. doi: 10.1093/nsr/nwaa036.

3. Organización Mundial de la Salud: Vías de transmisión del virus de la COVID-19: repercusiones para las recomendaciones relativas a las precauciones en materia de prevención y control de las infecciones. https: / / www.who.int/es/news room / commentaries/detail/modes-of transmission-of-virus-causing-covid-19implications-for-ipc-precautionrecommendations. (Accessed on June 22, 2020).

4. Wang W, Xu Y, Gao R. Detection of SARS-CoV-2 in Different Types of Clinical Specimens. JAMA 2020. 2020; 323(18): 1843-1844.

5. World Health Organization. Novel coronavirus situation reports -2. January 22, 2020. https: / / www. who.int/docs/default source/coronaviruse/situationreports/20200122-sitrep-2-2019-ncov.pdf (Accessed on June 22, 2020).

6. COVID-19 Treatment Guidelines Panel. Coronavirus Disease 2019 (COVID-19) Treatment Guidelines. National Institutes of Health. A v a i l a b l e https://www.covid19treatmentguidelines.nih.go $\mathrm{v} /$. (Accessed on June 22, 2020).

7. Jason $\mathrm{P}, \mathrm{Li} \mathrm{W}$, Lowell $\mathrm{L}$, et al. Intensive care management of coronavirus disease 2019 (COVID19): challenges and recommendations. Lancet Respir Med. 2020; 8: 506-17.

8. Alhazzani W, Møller MH, Arabi YM, et al. Surviving Sepsis Campaign: guidelines on the management of critically ill adults with Coronavirus Disease 2019 (COVID-19). Intensive Care Med. 2020;46(5):854-887. doi:10.1007/s00134-02006022-5

9. Russell CD, Millar JE, Baillie JK. Clinical evidence does not support corticosteroid treatment for 2019-nCoV lung injury. Lancet. 2020 doi: 10.1016/S0140-6736(20)30317-2.

10. Wu C, Chen X, Cai Y. Risk Factors Associated With Acute Respiratory Distress Syndrome and Death in Patients With Coronavirus Disease 2019 Pneumonia in Wuhan, China. JAMA Intern Med. $2020 ; 180(7): 934-943$. doi:10.1001/jamainternmed.2020.0994

11. Fadel R, Morrison AR, Vahia A, et al. Early Short Course Corticosteroids in Hospitalized Patients with COVID-19. Clin Infect Dis. 2020. doi:10.1093/cid/ciaa601

12. Cao J, Tu WJ, Cheng W, Yu L, Liu YK, Hu X, Liu Q.
Clinical Features and Short-term Outcomes of 102 Patients with Corona Virus Disease 2019 in Wuhan, China. Clin Infect Dis. 2020. doi: 10.1093/cid/ciaa243.

13. Wang D, Wang J, Jiang Q, et al. No Clear Benefit to the Use of Corticosteroid as Treatment in Adult Patients with Coronavirus Disease 2019: A Retrospective Cohort Study. medRxiv; 2020. DOI: 10.1101/2020.04.21.20066258.

14. Shang, Jin and Du, Ronghui and Lu, Qiaofa and Wu, Jianhong and $\mathrm{Xu}$, Shabei and $\mathrm{Ke}$, et al. The Treatment and Outcomes of Patients with COVID19 in Hubei, China: A Multi-Centered, Retrospective, Observational Study. Available at SSRN: https://ssrn.com/abstract $=3546060$ or http://dx.doi.org/10.2139/ssrn.3546060

15. Callejas-Rubio JL, Luna Del Castillo JD, de la Hera Fernández J, Guirao Arrabal E, Colmenero Ruiz M, Ortego Centeno N. Effectiveness of corticoid pulses in patients with cytokine storm syndrome induced by SARS-CoV-2 infection. Med Clin (Barc). 2020; 27:S0025-7753(20)30283-9. doi: 10.1016/j.medcli.2020.04.018.

16. Wang Y, Weiwei Jiang, Qi He, Cheng Wang, Baoju Wang, Pan Zhou, Nianguo Dong, Qiaoxia Tong. Early, low-dose and short-term application of corticosteroid treatment in patients with severe COVID-19 pneumonia: single-center experience from Wuhan, China. medRxiv 2020.03 .06 .20032342 ; d o i : https://doi.org/10.1101/2020.03.06.20032342

17. Yang $X, Y u Y, X u$ J. Clinical course and outcomes of critically ill patients with SARS-CoV-2 pneumonia in Wuhan, China: a single-centered, retrospective, observational study. The Lancet. $2020 ; \quad 8(5)$ : $\quad P 475-481$. DOI:https://doi.org/10.1016/S22132600(20)30079-5

18. Zhou F, Yu T, Du R, Fan G, Liu Y, Liu Z et al. Clinical course and risk factors for mortality of adult inpatients with COVID-19 in Wuhan, China: a retrospective cohort study. The Lancet. 2020; 395 , $\quad\left(\begin{array}{llll}1 & 0 & 2 & 2\end{array}\right)$. DOI:https://doi.org/10.1016/S01406736(20)30566-3

19. Zha L, Li S, Pan L, et al. Corticosteroid treatment of patients with coronavirus disease 2019 (COVID19). Med J Aust. 2020;212(9):416-420. doi:10.5694/mja2.50577

20. Xu J, Yang $X$, Yang L, et al. Clinical course and predictors of 60-day mortality in 239 critically ill patients with COVID-19: a multicenter retrospective study from Wuhan, China. Crit Care. 2020;24(1):394. doi:10.1186/s13054-020-03098-9

21. Horby P, Wei Shen Lim, Jonathan Emberson, Marion Mafham, Jennifer Bell, Louise Linsell, et al., RECOVERY Collaborative Group. Effect of Dexamethasone in Hospitalized Patients with COVID-19: Preliminary Report. medRxiv 
2020.06 .22 .20137273 ; d o i : https://doi.org/10.1101/2020.06.22.20137273

22. Corral L, Bahamonde A, de las Revillas FA, GomezBarquero J, Abadia-Otero J, Garcia-Ibarbia C, et., Glucocovid investigators. GLUCOCOVID: A controlled trial of methylprednisolone in adults hospitalized with COVID-19 pneumonia. medRxiv 2020.06 .17 .20133579 ; d o i : https://doi.org/10.1101/2020.06.17.20133579

23. Jeronimo CMP, Farias MEL, Val FFA, et al. Methylprednisolone as Adjunctive Therapy for Patients Hospitalized With COVID-19 (Metcovid): A Randomised, Double-Blind, Phase Ilb, PlaceboControlled Trial. Clin Infect Dis. 2020. doi:10.1093/cid/ciaa1177

24. Siemieniuk RA, Bartoszko JJ, Ge L, et al. Drug treatments for covid-19: living systematic review and network meta-analysis. BMJ. 2020;370:m2980. doi:10.1136/bmj.m2980

25. Ye Z, Wang Y, Colunga-Lozano LE, et al. Efficacy and safety of corticosteroids in COVID-19 based on evidence for COVID-19, other coronavirus infections, influenza, community-acquired pneumonia and acute respiratory distress syndrome: a systematic review and meta-analysis. CMA J . $2020 ; 192(27)$ : E 756 - E 767 . doi:10.1503/cmaj.200645

26. Yang Z, Liu J, Zhou Y, Zhao X, Zhao Q, Liu J. The effect of corticosteroid treatment on patients with coronavirus infection: a systematic review and meta-analysis. J Infect. 2020;81(1):e13-e20. doi:10.1016/j.jinf.2020.03.062

27. Lee $\mathrm{KH}$, Yoon S, Jeong $\mathrm{GH}$, et al. Efficacy of Corticosteroids in Patients with SARS, MERS and COVID-19: A Systematic Review and Meta-Analysis. J Clin Med. 2020;9(8):E2392. Published 2020 Jul 27. doi:10.3390/jcm9082392

28. Li H, Chen C, Hu F, et al. Impact of corticosteroid therapy on outcomes of persons with SARS-CoV-2, SARS-CoV, or MERS-CoV infection: a systematic review and meta-analysis. Leukemia. 2020;34(6):1503-1511. doi:10.1038/s41375-0200848-3.

\section{Correspondencia}

Edinson Dante Meregildo Rodriguez.

Dirección: Residencial Puertas del Sol, Calle 09, Mz. Y, Lt. 11; Cód. Postal 14007; Chiclayo; Lambayeque; Perú. Teléfono: 51944226126

Correo: dante meregildo@hotmail.com

\section{Revisión de pares}

Recibido: 22/09/2020

Aceptado: 20/11/2020 